\title{
Diagnostic utility of procalcitonin as a biomarker for late-onset neonatal sepsis
}

\author{
Chunmei Liu^, Chengzhi Fang, Lili Xie \\ Department of Neonatology, Renmin Hospital of Wuhan University and Hubei General Hospital, Wuhan, China \\ Contributions: (I) Conception and design: C Liu; (II) Administrative support: C Liu; (III) Provision of study materials or patients: All authors; (IV) \\ Collection and assembly of data: All authors; (V) Data analysis and interpretation: All authors; (VI) Manuscript writing: All authors; (VII) Final \\ approval of manuscript: All authors. \\ Correspondence to: Chunmei Liu. Department of Neonatology, Renmin Hospital of Wuhan University and Hubei General Hospital, Wuhan, China. \\ Email: liuchunmei123@126.com.
}

Background: To explore the diagnostic utility of procalcitonin (PCT) as a biomarker for late-onset neonatal sepsis (LONS).

Methods: The clinical and laboratory data of 131 neonatal patients in the neonatal intensive cares unit (NICU) of our center (Department of Neonatology, Renmin Hospital of Wuhan University) from June 1, 2015, to May 31, 2018, were retrospectively analyzed. These patients were divided into 3 groups based on their disease conditions: the bacterial sepsis (BS) group ( $=47)$, the fungal sepsis (FS) group ( $n=39)$, and the normal control group ( $\mathrm{n}=45$, without sepsis). Blood cultures, routine blood tests, and testing for PCT and C-reactive protein (CRP) were performed in all 3 groups. Both PCT and CRP were measured by using enzyme-linked immunosorbent assay (ELISA). Blood culture was performed in an automated blood culture system. Routine blood tests were performed by using a fully automatic hematology analyzer.

Results: Serum PCT level was significantly different between the BS group and control group $(\mathrm{P}<0.01)$ but showed no significant difference between the FS group and control group $(\mathrm{P}>0.05)$; the difference in CRP was statistically significant between the FS group and control group $(\mathrm{P}<0.01)$ but was not statistically significant between the BS group and control group $(\mathrm{P}>0.05)$. The areas under the receiver operating characteristics (ROC) curve were 0.979 and 0.826 for PCT/CRP in the BS group and FS group, with a best cutoff value of 0.93 and 33.27 , respectively; the sensitivities and specificities of PCT/CRP in these 2 groups were 0.962/0.679 and 0.964/0.964, respectively.

Conclusions: Compared with CRP, PCT is more sensitive in diagnosing BS but is not sensitive for diagnosing FS. Therefore, PCR is a useful biomarker in distinguishing BS from FS in neonates with lateonset sepsis.

Keywords: Procalcitonin (PCT); late-onset neonatal sepsis (LONS); diagnostic value

Submitted Mar 06, 2020. Accepted for publication Jun 07, 2020.

doi: $10.21037 / \mathrm{tp}-20-127$

View this article at: http://dx.doi.org/10.21037/tp-20-127

$\wedge$, ORCID: 0000-0001-2345-6789.

(C) Translational Pediatrics. All rights reserved. 


\section{Introduction}

Neonatal sepsis occurs during the first month (less than or equal to 28 days) after birth and may include infections at normally sterile sites and present other symptoms associated with the disease $(1,2)$. According to the age of onset, neonatal sepsis can be divided into early-onset neonatal sepsis (EONS) ( $\leq 72$ hours after birth) and late-onset neonatal sepsis (LONS) (>72 hours, except for late-onset Guillain-Barré syndrome) (2-4). Traditionally, both LONS and EONS are caused by bacterial infection; in recent years, however, an increasingly higher proportion of LONS cases have been attributed to fungal infections, which can involve focal symptoms in nerves and bone marrow and incur severe short- and long-term complications. Since the early clinical manifestations of neonatal sepsis are mild and atypical $(5,6)$, bacterial culture of sterile body fluids (SBF) remains the gold standard for the diagnosis of this disease; however, it is a non-sensitive and time-consuming technique. In contrast, real-time polymerase chain reaction for pathogenic microorganisms is expensive and has not been widely applied in clinical settings. Thus, a variety of auxiliary examinations including testing for biochemical markers and scoring systems have been developed to assist the selection of antibiotics in suspicious cases. Procalcitonin (PCT) is an early inflammatory factor produced during bacterial infections and has been widely acknowledged as a biomarker of bacterial infections in adult patients (7). PCT is more sensitive than C-reactive protein (CRP) in detecting bacterial infections but shows no significant difference in detecting fungal infections, and can prevent physiological interference for patients with LONS. Given these properties, PCT can be used as a diagnostic marker for LONS. It is particularly valuable when used in combination with CRP in patients with a negative blood culture for sepsis. EONS is mainly linked to mothers' perinatal factors and can be vertically transmitted. Thus, compared with CRP (which is more commonly used), PCT is more sensitive but more likely to be affected by the physiological factors of the neonates after birth; most notably, the PCT level dramatically rises within 72 hours after birth (and particularly within 48 hours). Therefore, the majority of studies have used PCT as a marker for ruling out infections (if it is negative), and few studies have used it as a diagnostic criterion (when it was positive) for EONS.

Therefore, this article proposes that, with the advances in neonatal intensive care, more LONS cases have been found to be caused by fungal infections, with their treatments being quite distinct from those for bacterial sepsis. Blood culture has many limitations, and its sensitivity is especially low for fungi. Consequently, our current study used PCT to compare the different findings between bacterial infections and fungal infections in neonatal patients with LONS in the neonatal intensive care unit (NICU), and attempted to identify different types of infections; in particular, we considered a blood culture showing negative results to indicate sepsis. An accurate and rapid clinical diagnosis is the basis for the successful treatment of sepsis, while identifying the infection type is crucial for selecting the rational treatments and alleviating the severe sequelae caused by sepsis (8). We present the following article in accordance with the STROBE reporting checklist (available at http://dx.doi.org/10.21037/tp-20-127).

\section{Methods}

The study follows the Helsinki Declaration and was approved by Ethics Committee of People's Hospital of Wuhan University (The ethics Committee of our school agreed but no number was given because there was no relevant project funding for this article). All the children in our department were treated with the informed consent of their guardians.

\section{Subjects}

The clinical and laboratory data of 131 neonatal patients aged $>72$ hours present in the NICU of our center from June 1, 2015, to May 31, 2018, were retrospectively analyzed. These patients were divided into three groups based on their disease conditions: a bacterial sepsis (BS) group ( $\mathrm{n}=47)$, a fungal sepsis (FS) group $(\mathrm{n}=39)$, and a normal control group $(n=45)$. These patients had negative blood culture results, negative infection markers with no clinical manifestations, and did not receive any antibacterial treatment during hospitalization. Since sepsis was suspected, PCT and CRP measurements and routine blood tests were performed in the BS group and FS group; in contrast, patients in the control group were premature infants who were ready to be discharged during the same period, in whom the possibilities of bacterial and fungal infections were ruled out. All the relevant tests were performed by the clinical laboratory in our center.

\section{Tests and instruments}

Routine blood tests and CRP measurement were performed 
Table 1 General data and clinical information of patients in the three groups

\begin{tabular}{|c|c|c|c|c|}
\hline Variables & BS group $(n=56)$ & FS group $(n=48)$ & Control group $(n=54)$ & $P$ value \\
\hline Gestational age (w) & $31.8 \pm 3.8$ & $30.9 \pm 4.2$ & $31.6 \pm 4.3$ & $>0.05$ \\
\hline Hospital stay (d) & $33.5 \pm 8.6$ & $34.9 \pm 7.5$ & $33.94 \pm 8.1$ & $>0.05$ \\
\hline
\end{tabular}

BS, bacterial sepsis; FS, fungal sepsis.

Table 2 PCT levels in the three groups

\begin{tabular}{lccr}
\hline Group & PCT $(\bar{x} \pm S D)$ & Pairwise comparison & t value \\
\hline BS group & $67.75 \pm 6.34$ & BS group vs. control group & 5.35 \\
FS group & $33.38 \pm 6.46$ & FS group vs. control group & 6.87 \\
Control group & $23.24 \pm 6.40$ & BS group vs. FS group & 1.59 \\
\hline
\end{tabular}

PCT, procalcitonin; BS, bacterial sepsis; FS, fungal sepsis.

using an automatic blood analysis system (XN-9000, Sysmex Co., Kobe, Japan). PCT was measured using electrochemical luminescence immunoassay (E601, Roche Cobas). Blood culture was completed in an automatic microbiological detection system (BacT/ALERT3D60, Bio Mérieux).

\section{Statistical analysis}

Statistical analysis was performed in the SPSS 19.0 software package (IBM, USA). The measurement data were tested for normality and homogeneity. Since these data were not normally distributed, so Kruskal-Wallis test based on rank statistic were applied for intergroup comparisons and pairwise comparisons. The receiver operating characteristic (ROC) curves were drawn, and the sensitivity, specificity, and areas under ROC curves (AUCs) were compared to evaluate the diagnostic performance of each indicator. A P value of $<0.05$ was considered statistically significant.

\section{Results}

\section{General data and clinical information}

There were no significant differences in age, weight, and gestational age among these 3 groups $(\mathrm{P}>0.05)$ (Table 1). Patients in the BS group and FS group met the following diagnostic criteria for sepsis (9): (I) with microorganisms identified in normally SBF after culture; or (II) with clinical manifestations plus 2 abnormal non-specific inflammation markers (Table 1).

\section{Results of PCT and CRP measurements in three groups}

The results of PCT and CRP measurements of the 3 groups are summarized in Tables 2, 3 .

\section{Areas under ROC curve in the three groups (Table 4)}

The results of areas under ROC curve in the 3 groups are shown in Figures 1,2 and Table 4.

The comparison between the BS group and FS group yielded areas under the PCT curve and CRP curve of 0.979 and 0.826 , respectively, with a sensitivity of 0.962 and 0.679 , and a specificity of 0.964 and 0.964 , respectively; thus, PCT was more sensitive than CRP for distinguishing BS from FS with the same specificity (the best cut-off values were 0.93 and 33.27). Comparison between the BS group and control group showed the areas under the PCT curve and CRP curve were 0.99 and 0.826 , respectively, with a sensitivity of 0.963 and 0.893 and a specificity of 1 and 1 , respectively; thus, PCT was more sensitive than CRP in identifying BS. The comparison between the FS group and control group yielded areas under the PCT curve and CRP curve of 0.679 and 0.772 , respectively, with a sensitivity of 0.714 and 0.536 , and a specificity of 0.593 and 0.963 , respectively; thus, PCT was less sensitive and more specific than CRP in identifying FS. Compared with CRP, PCT was more sensitive for BS but less sensitive for FS. 
Table 3 CRP levels in the three groups

\begin{tabular}{|c|c|c|c|c|}
\hline Group & $\operatorname{CRP}(\bar{x} \pm S D)$ & Pairwise comparison & t value & $P$ value \\
\hline BS group & $62.54 \pm 6.42$ & BS group vs. control group & 3.51 & $<0.05$ \\
\hline Control group & $22.56 \pm 6.35$ & BS group vs. FS group & 2.75 & $<0.05$ \\
\hline
\end{tabular}

CRP, C-reactive protein; BS, bacterial sepsis; FS, fungal sepsis.

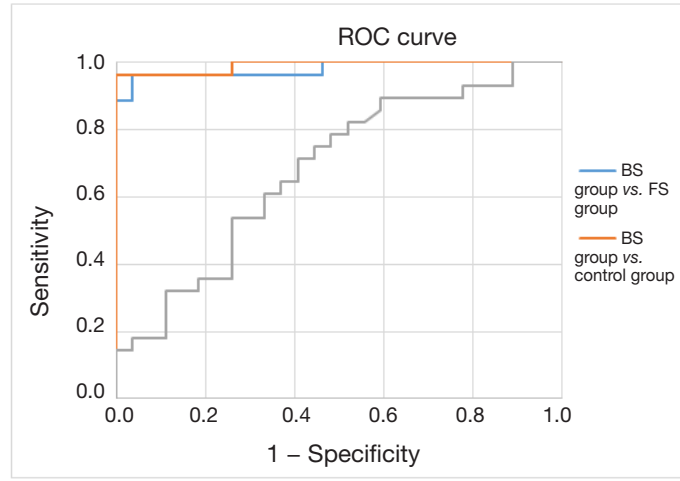

Figure 1 ROC curves of PCT. ROC, receiver operating characteristic; PCT, procalcitonin.

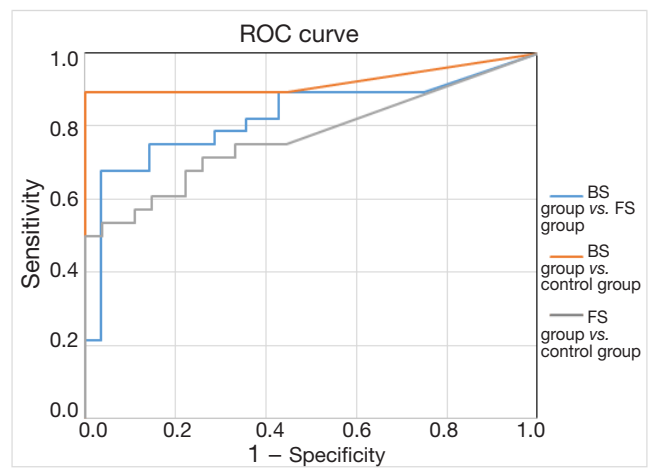

Figure 2 ROC curves of CRP. ROC, receiver operating characteristic; CRP, C-reactive protein.

\section{Discussion}

In addition to differing in the time of onset, EONS and LONS also differ in terms of etiology, clinical manifestations, treatment, and prevention measures. Unlike EONS (which is vertically transmitted), the pathogenesis of LONS is closely related to the colonization of pathogens and environmental factors, with preterm birth being its largest independent risk factor. The incidence of LONS is inversely proportional to the gestational age and can reach $25-30 \%$ in very low-birth-weight $(\leq 1,500 \mathrm{~g})$ infants $(1,10)$. In recent years, an increasingly higher proportion of LONS cases have been linked to fungal infections, which is quite different from the bacterial infections causing the EONS cases. In recent years, the treatment for EONS and for LONS have differed greatly. The treatment for EONS is based on epidemiological surveys on factors associated with vertical transmission and is typically empirical (mainly based on antibacterial treatment). In contrast, LONS, as a horizontal transmission, is associated mainly with the children's own microbial colonization and living environments; its treatment should be based on both the local common bacteria and the susceptibility to fungal infections (including factors such as gestational age, use of antibiotics, use of hormones, and intravenous catheterization). LONS patients, especially those with fungal infections, have higher case-fatality rates and casedisability rates, which is quite different from the rates in EONS cases caused by bacterial infections.

Due to certain limitations, including mainly the use of time-consuming and non-sensitive SBF culture for pathogens as the gold standard for diagnosis and the difficulty in collecting blood specimens from premature infants, a diagnosis of BS or FS often cannot be confirmed in a timely manner. Meanwhile, the occurrence and development of sepsis is associated with a series of inflammatory factors; among these is PCT, and it has been widely studied in adult and pediatric patients; compared with the traditional inflammatory markers (such as CRP and IL-6), PCT has shown superior diagnostic utilities in terms of sensitivity and specificity (11-13). Although many studies have investigated the value of PCT in diagnosing EONS, their conclusions were quite contradictory due to the impacts of various factors including physiological status and stress $(14,15)$. Furthermore, few relevant studies have been conducted on LONS. Therefore, our current study retrospectively explored the diagnostic value of PCT for LONS. 
Table 4 Analysis of areas under ROC curve in the three groups

\begin{tabular}{|c|c|c|c|c|c|c|}
\hline Pairwise comparison & Best cut-off values & Area & Sensitivity & Specificity & $95 \% \mathrm{Cl}$ & $P$ value \\
\hline BS group vs. control group & $0.854 / 8.265$ & $0.99 / 0.923$ & $0.963 / 0.893$ & $1 / 1$ & $0.969-1.0 / 0.836-1.0$ & $<0.05 /<0.05$ \\
\hline FS group vs. control group & $0.125 / 4.525$ & $0.679 / 0.772$ & $0.714 / 0.536$ & $0.593 / 0.963$ & $0.537-0.821 / 0.644-0.897$ & $<0.05 /<0.05$ \\
\hline
\end{tabular}

$\mathrm{ROC}$, receiver operating characteristic; BS, bacterial sepsis; FS, fungal sepsis.

As shown in Tables 2,3, pairwise comparisons among the BS group, FS group, and control group showed PCT was more sensitive than CRP in the diagnosis of BS, with a best cutoff value of $0.854 \mathrm{ng} / \mathrm{dL}$; in comparison, the cutoff value of PCT for diagnosing sepsis was $\geq 2 \mathrm{ng} / \mathrm{dL}$ in the ICU and NICU $(16,17)$. A lower cutoff value means higher sensitivity in the NICU. As shown in Table 4, the area under the ROC curve in distinguishing BS from FS was 0.979 and 0.826 for PCT and CRP, with a sensitivity of 0.962 and 0.679 , respectively. Thus, PCT was more sensitive than CRP in identifying BS and FS, with an optimal cutoff value of 0.93 . Its accuracy was also higher. PCT was not significantly different between the FS group and control group, suggesting PCT was neither sensitive nor specific in identifying fungal infection or FS.

Thus, PCT is more valuable than CRP in the diagnosis of late-onset BS but is not sensitive for identifying fungal infections. Since PCT has different sensitivity in diagnosing bacterial versus fungal infections, it can be used for the differential diagnosis of LONS. When a diagnosis of sepsis is suspected, serum PCT level may be detected. A serum PCT level of $\geq 0.93$ often suggests bacterial infection, whereas a serum PCT level of $<0.93$ is suggestive of fungal infection.

Since LONS occurs 72 hours after birth, serum PCT level is affected by perinatal and physiological factors. In our current study, comparison of PCT with CRP showed PCT had better biological diagnostic value for bacterial infection; since PCT showed significant differences in neonates with BS or FS, further analysis based on the area under ROC curve revealed that serum PCT level was a good biomarker for distinguishing bacterial infection from fungal infection. Therefore, based on clinical manifestations, PCT is more sensitive than CRP in detecting bacterial infections but shows no significant difference in detecting fungal infections; for patients with LONS, it can also avoid physiological interference. Therefore, PCT can be used as a diagnostic marker for LONS. It is particularly valuable when used in combination with CRP in patients with a negative result after blood culture for sepsis. For instance, if both CRP and PCT increase in an LONS patient, the possibility of bacterial infection can be considered; in contrast, if CRP increases but PCT shows no obvious change, a fungal infection will be considered. Antibacterial or antifungal therapies may be applied accordingly. Timely and rapid diagnosis and rational anti-infective treatment are critically important for lowering the case-fatality rate (18).

An initial diagnosis of LONS can be made based on clinical manifestations and the levels of both PCT and CRP. Accordingly, an antibacterial or antifungal treatment should be initiated as early as possible. Antibacterial treatment alone, as applied for EONS, may delay the treatment of fungal infection and worsen the disease condition. Early rational antifungal treatment will alleviate the severe complications and reduce the mortality in LONS patients. Despite these valuable findings, our current study was limited by its small sample size. Multicenter studies with large sample sizes are warranted to further support our findings and promote the application of PCT in clinical settings.

\section{Acknowledgments}

Funding: None.

\section{Footnote}

Reporting Checklist: The authors have completed the STROBE reporting checklist. Available at http://dx.doi. org/10.21037/tp-20-127

Data Sharing Statement: Available at http://dx.doi. org/10.21037/tp-20-127

Conflicts of Interest: All authors have completed the ICMJE uniform disclosure form (available at http://dx.doi. org/10.21037/tp-20-127). The authors have no conflicts of interest to declare. 
Ethical Statement: The authors are accountable for all aspects of the work in ensuring that questions related to the accuracy or integrity of any part of the work are appropriately investigated and resolved. The study follows the Helsinki Declaration and was approved by Ethics Committee of People's Hospital of Wuhan University (The ethics Committee of our school agreed but no number was given because there was no relevant project funding for this article). All the children in our department were treated with the informed consent of their guardians.

Open Access Statement: This is an Open Access article distributed in accordance with the Creative Commons Attribution-NonCommercial-NoDerivs 4.0 International License (CC BY-NC-ND 4.0), which permits the noncommercial replication and distribution of the article with the strict proviso that no changes or edits are made and the original work is properly cited (including links to both the formal publication through the relevant DOI and the license). See: https://creativecommons.org/licenses/by-nc-nd/4.0/.

\section{References}

1. Shane AL, Sanchez PJ, Stoll BJ. Neonatal sepsis. Lancet 2017;390:1770-80.

2. Greenberg RG, Kandefer S, Do BT, et al. Late-onset sepsis in extremely premature infants: 2000-2011. Pediatr Infect Dis J 2017;36:774-9.

3. Stoll BJ, Hansen N, Fanaroff AA, et al. Late-onset sepsis in very low birth weight neonates: the experience of the NICHD Neonatal Research Network. Pediatrics 2002;110:285-91.

4. Achten NB, van Meurs M, Jongman RM, et al. Markers of endothelial cell activation in suspected late onset neonatal sepsis in Surinamese newborns: a pilot study. Transl Pediatr 2019;8:412-418.

5. Das A, Shukla S, Rahman N, et al. Clinical indicators of late-onset sepsis workup in very low-birth-weight infants in the neonatal intensive care unit. Am J Perinatol 2016;33:856-60.

6. Quenot JP, Dargent A, Large A, et al. Treatment of sepsisinduced acute kidney injury in the ICU: the therapeutic targets do not seem to be established yet. Ann Transl Med 2019;7:S181.

7. van Rossum AM, Wulkan R, Oudesluys-Murphy A. Procalcitonin as an early marker of infection in neonates and children. Lancet Infect Dis 2004;4:620-30.
8. Lin KH, Wang FL, Wu MS, et al. Serum procalcitonin and Creactive protein levels as markers of bacterial infection in patients with liver cirrhosis: a systematic review and meta-analysis. Diagn Microbiol Infect Dis 2014;80:72-8.

9. Ganesan $P$, Shanmugam $P$, Sattar SB, et al. Evaluation of IL-6, CRP and hs-CRP as early markers of neonatal sepsis. J Clin Diagn Res 2016;10:DC13-7.

10. Gowda H, Norton R, White A, et al. Late-onset neonatal sepsis - a 10-year review from North Queensland, Australia. Pediatr Infect Dis J 2017;36:883-8.

11. Luzzani A, Polati E, Dorizzi R, et al. Comparison of procalcitonin and $\mathrm{C}$-reactive protein as markers of sepsis. Crit Care Med 2003;31:1737-41.

12. Müller B, Becker KL, Schachinger H, et al. Calcitonin precursors are reliable markers of sepsis in a medical intensive care unit. Crit Care Med 2000;28:977-83.

13. Selberg O, Hecker H, Martin M, et al. Discrimination of sepsis and systemic inflammatory response syndrome by determination of circulating plasma concentrations of procalcitonin, protein complement $3 \mathrm{a}$, and interleukin-6. Crit Care Med 2000;28:2793-8.

14. Hedegaard SS, Wisborg K, Hvas AM. Diagnostic utility of biomarkers for neonatal sepsis - a systematic review. Infect Dis (Lond) 2015;47:117-24.

15. Bhandari V. Effective biomarkers for diagnosis of neonatal sepsis. J Pediatric Infect Dis Soc 2014;3:234-45.

16. Erickson YO, Samia NI, Bedell B, et al. Elevated procalcitonin and C-reactive protein as potential biomarkers of sepsis in a subpopulation of thrombotic microangiopathy patients. J Clin Apher 2009;24:150-4 .

17. O'Grady NP, Barie PS, Bartlett JG, et al. Guidelines for evaluation of new fever in critically ill adult patients: 2008 update from the American College of Critical Care Medicine and the Infectious Diseases Society of America. Crit Care Med 2008;36:1330-49.

18. Carrol ED, Thomson A, Hart C. Procalcitonin as a marker of sepsis. Int J Antimicrob Agents 2002;20:1-9.

(English Language Editor: John Gray, AME Publishing Company)

Cite this article as: Liu C, Fang C, Xie L. Diagnostic utility of procalcitonin as a biomarker for late-onset neonatal sepsis. Transl Pediatr 2020;9(3):237-242. doi: 10.21037/tp-20-127 